\title{
Outcome of Early vs. Late Antibiotic Administration in Management of Patients with Severe Sepsis
}

\section{Shaheer Zahid}

Internal Medicine, Jackson Park Hospital and Medical centre, Chicago, IL, USA

${ }^{*}$ Corresponding author: Shaheer Zahid, Attending Physician, Board Certified, Internal Medicine, Jackson Park Hospital and Medical centre, Chicago, IL, USA, Tel: (773) 947-7500; E-mail: sh.zahid88@gmail.com

Received date: Sep 10, 2018; Accepted date: Sep 22, 2018; Published date: Sep 28, 2018

Copyright: $@ 2018$ Zahid S. This is an open-access article distributed under the terms of the Creative Commons Attribution License, which permits unrestricted use, distribution, and reproduction in any medium, provided the original author and source are credited.

\begin{abstract}
Sepsis is a potentially life-threatening complication of an infection. Sepsis occurs when chemicals are released into the bloodstream to fight the infection triggering inflammatory responses throughout the body. This inflammation can trigger a cascade of changes that can damage multiple organ systems, causing them to fail. With the increasing population at risk of sepsis in the United States, it has become crucial to recognize signs and symptoms of sepsis; also important is to refine and develop guidelines that will reduce the mortality.

This study focused on the patients of Jackson Park hospital in the ER/ICU/Floors between Jan 1, 2014, and Dec 31,2014 , and compared the appropriateness of antibiotics, their administration time, microbial isolate and site of infection to the mortality rate seen amongst the patients. All the collected data were compared using tables, pie chart and bar graphs. The study showed patients receiving antibiotics within 3 hours of arrival to the ED had a reduction in mortality by $49 \%$ with sepsis from $E$. coli, MRSA and other Staph infections were among the most common infections leading to mortality among the patients. Lungs and UTI infections were the most common site, with respiratory tract having $27 \%$ mortality among all patients presenting with sepsis.
\end{abstract}

The study allowed a prompt evaluation of sepsis screening protocol, as well as developing new protocols to include certain antibiotics readily available in the ED and antibiogram to be done for all sepsis patients.

Keywords: Sepsis; Antibiotic administration; Microbial isolate from infection; Site of infection; The mortality rate in sepsis; Organ failure from sepsis

\section{Introduction}

In 1992, the American College of Chest Physicians (ACCP) and the Society of Critical Care Medicine (SCCM) introduced definitions for systemic inflammatory response syndrome (SIRS), sepsis, severe sepsis, septic shock, and multiple organ dysfunction syndrome. SIRS is defined as a patient having 2 or more of the following variables: Fever of more than $38^{\circ} \mathrm{C}\left(100.4^{\circ} \mathrm{F}\right)$ or less than $36^{\circ} \mathrm{C}\left(96.8^{\circ} \mathrm{F}\right)$, Heart rate of more than 90 beats per minute, Respiratory rate of more than 20 breaths per minute or arterial carbon dioxide tension $\left(\mathrm{PaCO}_{2}\right)$ of less than $32 \mathrm{~mm} \mathrm{Hg}$, Abnormal white blood cell count $(>12,000 / \mu \mathrm{L}$ or $<$ $4,000 / \mu \mathrm{L}$ or $>10 \%$ immature \{band $\}$ forms) [1]

In 2001, the definition of sepsis and septic shock were redefined to include the new understanding of pathophysiology seen with the advancement in technology. Under the new definition, sepsis is a lifethreatening organ dysfunction caused by a dysregulated host response to infection and Septic shock is defined as a subset of sepsis in which underlying circulatory and cellular metabolism abnormalities are profound enough to substantially increase mortality [2].

Treatment for patients in later stages who require critical care necessitates organ support and prevention of nosocomial infection. It is crucial to determine the likely source of the infection, and administer intravenous (IV) empiric antimicrobial agents until culture results become available, at which point more narrow-spectrum agents can be used. The goal of early administration of broad-spectrum antibiotic is to eradicate the infection, reduce morbidity, and prevent complications, but the appropriate choice of antibiotic can play a huge role in the outcome [3].

The US Center for Disease Control's National Center for Health Statistics estimates that, based upon information collected for billing purposes, the number of times people were in the hospital with sepsis increased from 621,000 in the year 2000 to $1,141,000$ in 2008 [4]. With the increase in the aging population, infection with treatment-resistant microbes and people getting more procedures, immunosuppressive drugs, chemotherapy, and organ transplants; the cases of sepsis are increasing dramatically. Based on Mayr FB, et al. research in 2014 [3], severe sepsis is a leading cause of death in the United States and the most common cause of death among critically ill patients in noncoronary intensive care units (ICU). Surviving sepsis campaign (SCC) international consensus guidelines recommends initiating broadspectrum therapy within the first hour of severe sepsis or septic shock. But recent data demonstrated that only $68 \%$ of patients received broad-spectrum antibiotics within the first 3 hours of Emergency Department presentation [1].

The purpose of this work is to determine the effect of early versus late antibiotic administration on the outcome of patients presenting at Jackson Park hospital ER/ICU/ with symptoms of severe sepsis. Based new 2015 update of International guidelines for management of severe sepsis and septic shock three hours were used as a dividing line among early and late administration of antibiotics $[5,6]$. Based on previous studies [7], it can be hypothesized that early antibiotic treatment can have a drastic positive impact on the future outcome for the patient; 
Page 2 of 4

though the results can vary depending on the microbes causing the infection and the resistance to the antibiotics being used, we still predicted a reduction in mortality related to early treatment.

\section{Methods}

A retrospective study was conducted focusing on comparing the outcome of early versus late antibiotic administration in the management of patients with severe sepsis. The data were collected using 100 or more charts of patients aged eighteen and above with a diagnosis of sepsis, presenting at Jackson Park hospital ER/ICU/Floors between Jan 1, 2014, and Dec 31, 2014.

The patients included in the study were suspected or confirmed bacterial sepsis. Severe sepsis was defined as the presence of symptomatic bacteremia in conjunction with the systemic inflammatory response syndrome (SIRS) and complicated by organ dysfunction. Included were also patients with septic shock, defined as a case of sepsis with underlying circulatory, cellular, and metabolic abnormalities.

The timing was defined as reported time to antibiotic administration in relation to emergency department triage and/or the recognition of sepsis/septic shock. Early antibiotic therapy was defined as a therapy within three hours and late therapy as therapy after 3 hours. A comparison was done to see the ratio of survival among the population receiving antibiotics before 3 hours vs after 3 hours. The mortality among patient with different sites of infection was compared to the ratio of survival in patients' with infection at the site; this comparison allowed us a better understanding of the impact such factors have on the survival of septic patients.

Patient charts with lack of antibiotic timing data were excluded, also patients who were younger than 18 years, immune-compromised and no registered patient outcome regarding their management and diagnosis.

The patient outcome after their diagnosis of sepsis was compared to prophylaxis antibiotics given and administration time. Also, the mortality rate, microbial isolate, and site of infection were compared using tables, pie chart and bar graphs.

The limitations to the study could be: having limited statistical input (sample size calculation, p-value), inconsistent charting in regards to time of the administration of antibiotics, inconsistent availability of culture reports, culture sites and comorbidities that were not reported and/or adjusted for.

\section{Results}

\begin{tabular}{|c|c|c|c|c|}
\hline \multicolumn{2}{|c|}{} & \multicolumn{2}{|c|}{ Expired } & \multirow{2}{*}{ Total } \\
\cline { 2 - 4 } & Yes & No & \multirow{2}{*}{} \\
\hline \multirow{2}{*}{ Time Abx Adim } & $\leq 3$ hour & 9 & 42 & 51 \\
\cline { 2 - 5 } & $>3$ hour & 21 & 37 & 58 \\
\hline Total & & 30 & 79 & \\
\hline
\end{tabular}

Table 1: Time of administration of antibiotics.

Based on the results obtained from 109 patient charts; mortality from sepsis was $27.52 \%(30 / 109)$ overall, with $17.65 \%$ (9/51) in those patients treated within 3 hour and $36.21 \%(21 / 58)$ in those receiving treatment after 3 hour; time zero was considered as the time arrived in the emergency department.

Table 1 compares the mortality rate among septic patient receiving the antibiotic within 3 or more than 3 hour of admittance to the Jackson Park hospital ER/ICU/Floors.

The results of the site of the culture are presented in Figure 1. The results demonstrated that most culture results did not grow any organism $(24.49 \%)$ or the lab was unable to identify them. But amongst the cultured microbes E. coli $(8.16 \%), M R S A(7.48 \%)$ and other Staph infections were the most common microbes found in patients presenting to the emergency room and internal medicine floor; while Pseudomonas and Acinetobacter were more commonly seen in ICU patients.

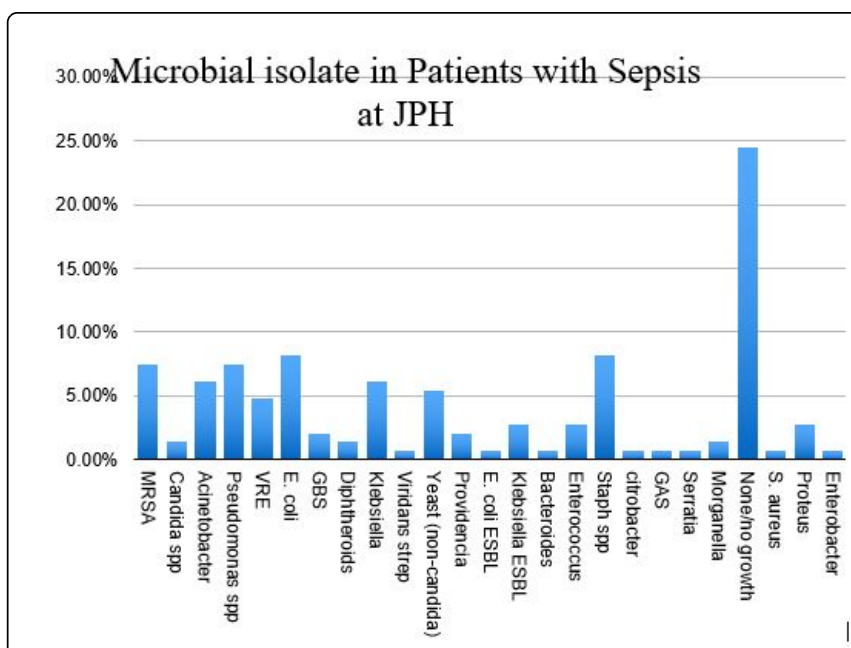

Figure 1: The figure compares the microbial isolate in patients with sepsis at Jackson Park hospital.

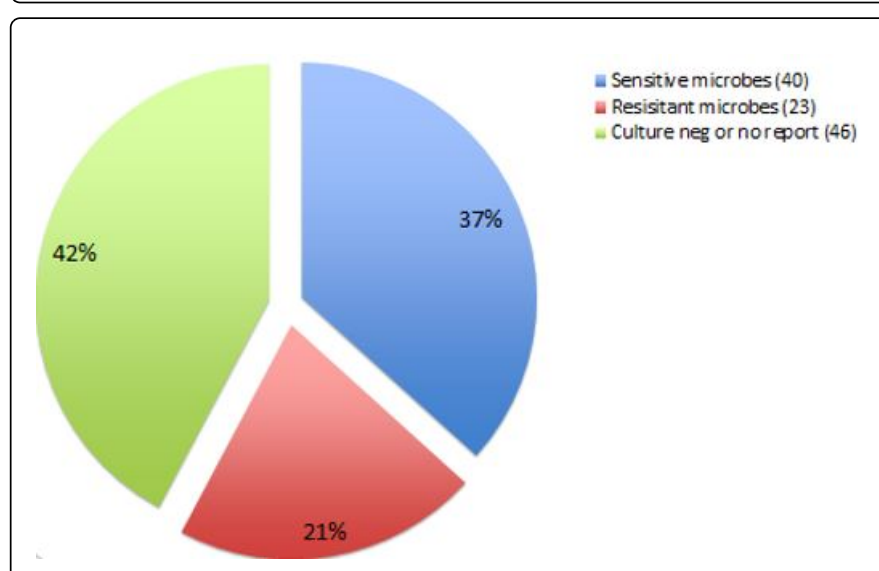

Figure 2: Appropriateness of Antibiotics.

Figure 2 compares the appropriateness of prophylactic antibiotic therapy among the patients presenting to Jackson park hospital emergency room. The data suggested that $42 \%(46 / 109)$ of the microbes could not be cultured or produced no report, while $37 \%$ (40/109) of patients received appropriate antibiotics because isolates were sensitive to the antibiotics they received; and 21\% (23/109) had microbes that were resistant to the initial treatment. 
Figure 3 compares the mortality among the patients that were infected with microbes sensitive to antibiotic versus microbes resistant to initial antibiotic treatment. Mortality was $7 \%$ when microbes were sensitive to the antibiotic and $39 \%$ when microbes were resistant.

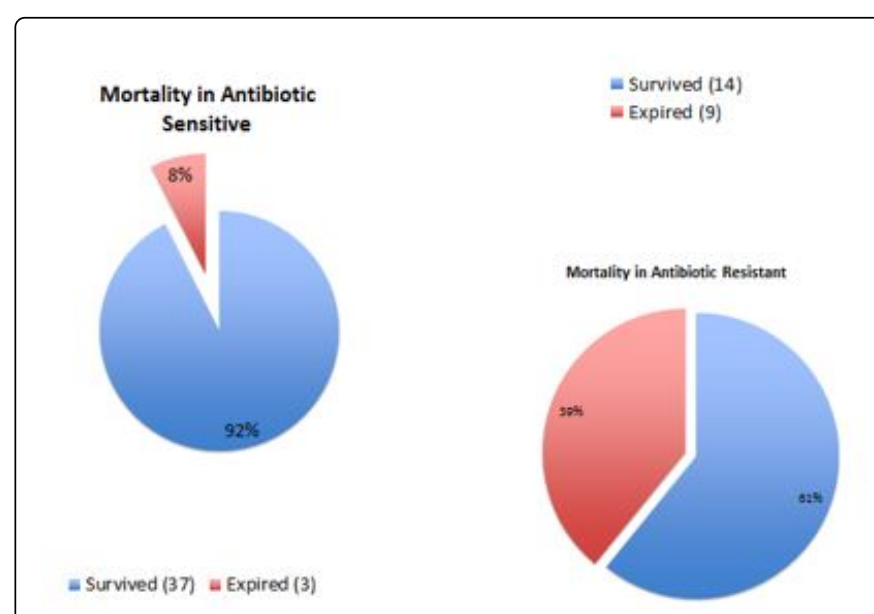

Figure 3: Comparison among antibiotic sensitive vs. antibiotic resistant microbes among septic patients.

Figures 4 and 5 compared the most common site of infection among septic patients presenting to Jackson Park hospital. The most common site of infection noted was the respiratory tract with a $27 \%$ mortality rate, and urinary tract being the second most common infection site with $17.6 \%$ mortality rate.

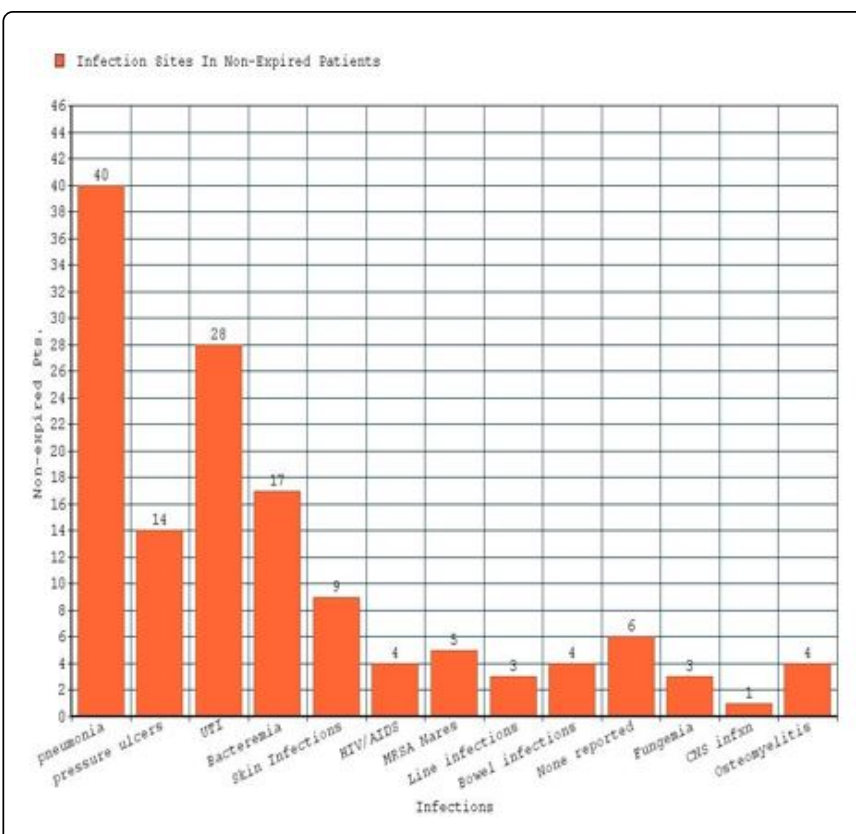

Figure 4: Infection sites of surviving patients presenting with sepsis.

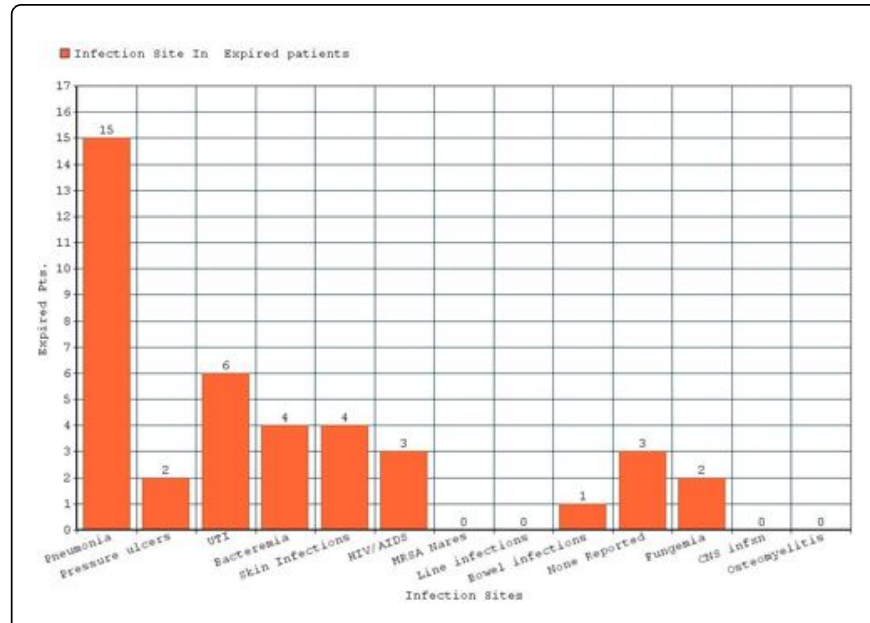

Figure 5: Infection sites of expired patients presenting with sepsis.

\section{Discussion}

\section{Time of antibiotic administration}

By using three hours as a dividing line among the presenting patients at Jackson Park Hospital, presented in table 1, we can clearly see the decrease in mortality when antibiotics are administered early within three hours of the presentation of the patient to the hospital. It is in accordance with the research done by Gaieski et al. [8]: a delay in the approach to the administration of appropriate antimicrobials is the primary determinant of mortality in patients with severe sepsis and septic shock. There are many different types of infection which can lead to sepsis; with pneumonia, urinary tract infection and skin infection being among the common one. According to the Centers for Disease Control and Prevention (CDC) severe burn or wound, people with weakened immune systems, babies, very young children, the elderly or people with chronic illnesses are most at risk.

\section{Mortality related to microbe isolate and antibiotic sensitivity}

In order to reduce organ damage antibiotics need to be given as early as possible; though without knowing the exact microbe and its sensitivity, the infection can become difficult to treat. In cases where initial broad-spectrum antibiotics are not effective, a microbe isolate and antibiotic sensitivity allow us to determine the best treatment option for the patient. In clinical setting, white blood count serves as an important factor for knowing the antibiotic sensitivity for a microbe; but Kirby-Bauer method is mostly used when understanding specific sensitivity of most microbes. The Delayed treatment can lead to sepsis-causing more damage to body organs which can greatly reduce a patient survival rate. Since microbe isolate and antibiotic sensitivity have a big impact on patient mortality, it is an important variable to consider. Data collected from the patients of Jackson Park hospital showed that most of the microbe cultures had no growth; in cases where growth was seen, E. coli, MRSA and other Staph infections were among the most common to microbes. As seen by Sara E. Cosgrove [9], there is an association between the development of antimicrobial resistance in Staphylococcus aureus, Enterococci, and gram-negative bacilli and increases in morbidity and mortality. For many patients, inadequate or delayed therapy and severe underlying 
Page 4 of 4

disease are primarily responsible for the adverse outcomes of infections caused by antimicrobial-resistant organisms. Strategies to prevent antibiotic resistance and spread of antimicrobial-resistant organisms are essential. One limiting factor of the study was bacterial culture technique; since most of the patient presenting with sepsis are treated in an emergency room setting, procedure set in place were followed, that included taking blood samples, and giving patients broad spectrum antibiotic treatment. Meanwhile, pathology results were obtained from the lab which was available to us at the hospital, but since patients were presenting in an emergency setting, cultures would be studied on a short term basis by the pathology lab leading to majority of the patient's lab result showing no bacterial growth or identification. By reducing the sample size for our study, our overall margin of error was increased. Another cause of bias amongst our study could be bacterial colonization amongst patients; since most of the patients were presenting to the same emergency room. Even though all protocol was followed, there is always a chance of spread of same infection. In the future we would like have a stronger focus on our lab work, to get better results in order to reduce the margin of error probably seen within the study.

\section{Morality based on the site of infection}

Based on Gaieski D et al. there is a direct correlation with site of infection and patients mortality [8]. Our data as presented in Figure 5 shows, how the two main sites of infection with the highest mortality were lung and urinary tract. Co-incidently lower respiratory and urinary tract are among the most difficult site to obtain an uncontaminated culture from, due to their anatomy and normal flora of the tracks; this causes a delay in treating the underlying infection in these sites which leads to a higher mortality rate among the patient population.

\section{Conclusion}

Based on our study, it can be concluded that patients in Jackson park hospital receiving antibiotics within 3 hours of arrival to the ED had a reduction in mortality by $49 \%$; lungs and UTI infections being the most common site with respiratory tract having $27 \%$ mortality among all patients. It is recommended that guidelines should be developed that allow for early recognition of the signs of sepsis, administration of antibiotics and consideration of the site of the infection and the likely microbes when deciding on the choice of the antibiotic.

\section{Acknowledgments}

The author thanks Dr. Branka Filipovic and Dr. Ali Syed for their excellent assistance. There was no source or sources of funding used to assist in writing this research thesis.

\section{References}

1. Bone RC, Balk RA, Cerra FB, Dellinger RP, Fein AM, et al. (1992) Definitions for sepsis and organ failure and guidelines for the use of innovative therapies in sepsis. The ACCP/SCCM Consensus Conference Committee. American College of Chest Physicians/Society of Critical Care Medicine. Chest 101: 1644-1655.

2. Singer M, Deutschman CS, Seymour CW, Shankar-Hari M, Annane D, et al. (2016) The third international consensus definitions for sepsis and septic shock (Sepsis-3). JAMA 315: 801-810.

3. Mayr FB, Yende S, Angus DC (2014) Epidemiology of severe sepsis. Virulence 5: 4-11.

4. Hall MJ, Williams SN, DeFrances CJ, Golosinskiy A (2011) National Center for Health Statistics Data Brief No. 62 June 2011. Inpatient care for septicemia or sepsis: a challenge for patients and hospitals.

5. Bone RC (1994) Sepsis and its complications: the clinical problem. Crit Care Med 22: S8-11.

6. Dellinger RP, Levy MM, Rhodes A, Annane D, Gerlach H (2013) Surviving Sepsis Campaign Guidelines Committee including the Pediatric Subgroup. Surviving sepsis campaign: international guidelines for management of severe sepsis and septic shock: 2012. Crit Care Med 41: 580-637.

7. Russell JA, Singer J, Bernard GR, Wheeler A, Fulkerson W, et al. (2000) Changing pattern of organ dysfunction in early human sepsis is related to mortality. Crit Care Med 28: 3405-3411.

8. Gaieski DF, Mikkelsen ME, Band RA, Pines JM, Massone R, et al. (2010) Impact of time to antibiotics on survival in patients with severe sepsis or septic shock in whom early goal-directed therapy was initiated in the emergency department. Crit Care Med 38: 1045-1053.

9. Sara E (2006) The relationship between antimicrobial resistance and patient outcomes: mortality, length of hospital stay, and health care costs. Clin Infect Dis 2: S82-S89. 